\title{
SOME SPACES RELATED TO TOPOLOGICAL INEQUALITIES PROVEN BY THE ERDÖS-RADO THEOREM
}

\author{
WILLIAM G. FLEISSNER
}

\begin{abstract}
The Erdös-Rado theorem is very useful in proving cardinal inequalities in topology. It has been suggested that certain of these inequalities might be strengthened. We note that trees constructed by Jensen and Gregory using various extra axioms of set theory yield several counterexamples to these suggestions; for example, a space $X,|X|=\omega_{2}, c(X)=\omega_{1}$, $\chi(X)=\omega$, answering a question of Hajnal and Juhász. We consider the apparently similar relation between $|X|, e(X)$, and $d \Psi(X)$ of Ginsburg and Woods. Using combinatorial consequences of $V=L$, we construct $G_{\delta}$ tree families, and establish that, assuming $V=L$, an infinite cardinal $\kappa$ is weakly compact iff $d \Psi(X)<\kappa, e_{a}(X) \subset \kappa$ imply $|X|<\kappa$.

We consider products of countable chain condition spaces, and show that, using Cohen forcing that $\left(2^{\omega}\right.$ can be anything allowed by König's theorem and there are spaces $\left.X, Y, c(X)=c(Y)=\omega, c(X \times Y)=2^{\omega}\right)$. A variation is a space $W$ with the property $c\left(W^{n}\right)=\omega_{n-1}$.
\end{abstract}

The Erdös-Rado theorem, $\left(2^{\kappa}\right)^{+} \rightarrow\left(\kappa^{+}\right)_{\alpha}^{2}, \quad \alpha<\kappa$, is very useful in establishing inequalities of cardinal functions, as amply illustrated in I. Juhász book. It has been suggested that certain of these inequalities might be sharpened. For example, $|X| \leqslant 2^{x(X) c(X)}$ gives a bound for $|X|$ in terms of the single variable $\max (\chi(X), c(X))$. Can a sharper bound be given as a function of two variables, $\chi(X)$ and $c(X)$ ? The product of $k$ copies of the two point discrete space demonstrates that $\chi(X)$ must be in the exponent, but no example requiring $c(X)$ to be in the exponent is known. The starting point of this research was the conjecture that $|X| \leqslant c(X)^{x(X)}$.

In A-E below, we list five inequalities proven by the Erdös-Rado theorem. $A, B$, and $C$ appear in $[\mathbf{J u}], D$ in $\left[\mathbf{G W}_{1}\right]$ and $E$ in $\left[\mathbf{G W}_{2}\right]$. We also list how certain spaces, whose existence is known to be consistent with the usual axioms of set theory, refute conjectured improvements. $B$ is a first countable $\omega_{2}$-Souslin line, $L$ is the order compactification of $B, S$ is the disjoint union of $\omega$ copies of $L$, and $A$ is the antichain in an $\omega_{2}, \omega G_{\delta}$ tree family.

A. $|X| \geqslant 2^{x(X) c(X)}$ for $T_{2}$ spaces $X$ : our space $B$ refutes $|X| \leqslant c(X)^{x(X)}$.

B. $|X| \leqslant 2^{\psi(X) s(X)}$ for $T_{2}$ spaces $X$ : our space $B$ refutes $|X| \leqslant s(X)^{\psi(X)}$.

C. $c_{\omega}(X) \leqslant 2^{c(X)}$ for compact $T_{2}$ spaces $X$; our space $L$ refutes $c_{\omega}(X) \leqslant$ $c(X)^{\omega}$.

Received by the editors March 12, 1977 and, in revised form, December 16, 1977.

AMS (MOS) subject classifications (1970). Primary 54A25, 04A20.

Key words and phrases. Cardinal functions in topology, trees, $V=L$, Erdös-Rado theorem, cellularity, character, spread, $G_{\delta}$ diagonal, products of ccc spaces, Cohen forcing. 
D. $c(\beta X-X) \leqslant 2^{K(X) c(X)}$ for completely regular spaces $X$; our space $S$ refutes $c(\beta X-X) \leqslant c(X)^{K(X)}$.

E. $|X| \leqslant 2^{d \psi(X) e(X)}$ for $T_{1}$ spaces $X$; our space $A$ refutes $|X| \leqslant e(X)^{d \psi(X)}$.

The plan of this paper is as follows. In $\S 1$ we introduce the notation and terminology used throughout this paper. We define convenient trees and use them to construct the spaces $B, L$ and $S$. Next we define $G_{\delta}$ tree families and use them to define the space $A$. In $\S 3$, we construct convenient trees from Souslin trees and construct $G$ tree families assuming $V=L$. Then we discuss a $\sup =\max$ problem related to inequality $\mathrm{E}$ and contrast it with the analogous problems A-D. In $\S 5$, we discuss the cellularity of the product of two ccc spaces. The Erdös-Rado theorem gives an upper bound of $c$ and we show that this upper bound can be attained no matter what the value of $c$.

1. We denote the cardinality of $X$ by $|X|$. We use $[\alpha]^{\beta}$ to denote the set of subsets of $\alpha$ of cardinality $\beta:[\alpha]^{<\omega}$ is the set of finite subsets of $\alpha$. The partition relation $\alpha \rightarrow(\beta)_{\gamma}^{n}$ means that whenever $P:[\alpha]^{n} \rightarrow \gamma$ there is $\delta \in \gamma$ and $H \in[\alpha]^{\beta}$ with $P^{\prime \prime}[H]^{n}=\{\delta\}$.

For a topological space $X$, with topology $\sigma(X)$, we define

\begin{tabular}{|c|c|}
\hline cellularity & $c(X)=\omega \cdot \sup \{|\mathscr{Q}|: \mathscr{Q} \subset \sigma(X), \mathscr{Q}$ is disjoint $\}$ \\
\hline$G_{\delta}$ cellularity & $c_{\omega}(X)=\omega \cdot \sup \left\{|\mathscr{Q}|: U \in \mathscr{Q}\right.$ is $G_{\delta}, \mathscr{Q}$ is disjoint $\}$ \\
\hline character & $\chi(X)=\sup \left\{\min \left\{\left|\mathscr{Q}_{p}\right|: \mathscr{Q}_{p}\right.\right.$ is a nghd basis at $\left.p\right\}:$ \\
\hline & $p \in X\}$ \\
\hline pseudocharacter & $\psi(X)=\sup \left\{\min \left\{\left|\mathscr{U}_{p}\right|: \cap \mathscr{U}_{p}=\{p\}\right\}: p \in X\right\}$ \\
\hline spread & $s(X)=\omega \cdot \sup \{|D|: D \subset X, D$ is discrete $\}$ \\
\hline extent & $e(X)=\omega \cdot \sup \{|D|: D \subset X, D$ is closed, discrete $\}$ \\
\hline$G_{\delta}$ degree & $d \psi(X)=\min \left\{|\mathscr{Q}|: \mathscr{Q} \subset \sigma\left(X^{2}\right), \cap \mathcal{U}=\operatorname{diag} X\right\}$ \\
\hline$K$ coweight & $K(X)=\min \{|\mathscr{K}|: K \in \mathscr{K}$ is cpt, $\forall H \subset X$, \\
\hline
\end{tabular}

$$
H \text { cpt } \exists K \in \mathscr{K}, H \subset K\} \text {. }
$$

We define (the phrase) $c_{a}(X)<\kappa$ to mean that there is no set of cardinality $\kappa$ of disjoint open sets of $X$. We similarly define $e_{a}(X)<\kappa$ and $s_{a}(X)<\kappa$. For a completely regular space $X, \beta X$ is the Stone-Cech compactification. $X$ is a ccc space iff $c(X)=\omega$.

A tree is a partially ordered set $T$, such that for every $x \in T$, the set $\hat{x}=\{y \in T: y<x\}$ is well ordered. Set $\bar{x}=\{x\} \cup \hat{x}$. The order $o(x)$ of $x$ is the order type of $\hat{x}$. The $\alpha$ th level of $T$ is $T_{\alpha}=\{x \in T: o(X)=\alpha\}$. The restriction of $T$ to height $\alpha$ is

$$
T \mid \alpha=\{x \in T: o(x)<\alpha\} .
$$

A branch of $T$ is a maximal linearly ordered subset of $T$; an $\alpha$-branch is a branch of order type $\alpha$. We say an $\alpha$-branch $b$ of $T \mid \alpha$ continues if there is $x \in T_{\alpha}, \hat{x}=b$. An antichain of $T$ is a set of incomparable elements of $T$; we 
abbreviate maximal antichain by mac. A $\lambda$-Souslin tree is a tree with elements of order $\alpha$ for $\alpha<\lambda$, but no branches or antichains of cardinality $\lambda$.

2. Problem 77/A of $[\mathbf{E H}]$ is whether $c(X)=\omega_{1}$ implies $|X|=\omega_{1}$ for first countable linearly ordered spaces $X$, assuming the Continuum Hypothesis. This is a special case of the question of whether inequality $\mathrm{A}$ can be sharpened. We begin this section by reviewing Jensen's solution to this problem.

Let us call a tree $T \lambda, \mu$ convenient if

(i) every antichain of $T$ has cardinality less than $\lambda$;

(ii) every element of $T$ has an infinite number of immediate successors;

(iii) there are at least $\lambda$ branches of $T$ of cofinality $\mu$.

Let $T$ be a $c^{+}, \omega$ convenient tree; let $B=\left\{b_{\alpha}: \alpha<c^{+}\right\}$be a family of branches of cofinality $\omega$; let $\left\{x_{\alpha}^{n}: n<\omega\right\}$ be $<_{T}$ increasing and cofinal in $b_{\alpha}$.

We obtain our spaces by "squashing" $T$ to a line $\left[\mathbf{K p}_{\mathbf{1}}\right]$, [Ru]. Let $<_{0}$ be a linear order on $T$. Because of (ii) we may choose $<_{0}$ so that for all $x \in T$, the set of immediate successors of $x$ has neither a $<_{0}$ greatest nor a $<_{0}$ least element. We define a linear order $<_{1}$ on $\operatorname{Br}(T)$, the set of all branches of $T$, by

$$
b<_{1} b^{\prime} \text { iff } \exists \alpha b\left|\alpha=b^{\prime}\right| \alpha \text { and } b(\alpha)<_{0} b^{\prime}(\alpha) .
$$

We give $\operatorname{Br}(T)$ the topology induced by the order $<_{1}$. Let $B\left(=\left\{b_{\alpha}\right.\right.$ : $\left.\alpha<c^{+}\right\}$) have the subspace topology. Let $L$ be the order completion of $\operatorname{Br}(T)$ with endpoints. By the Heine-Borel theorem, $L$ is compact. Then $L \times \omega$, (i.e. countably many disjoint copies of $L$ ) is $\sigma$-compact.

For $x \in T$, define $(x)=\{b \in \operatorname{Br}(T): x \in b\}$. The point of (ii) and our choice of $<_{0}$ is that $(x)$ is open in $\operatorname{Br}(T)$, and in $L,(x) \subset$ Interior (closure $(x))$. The point of (i) is that $c(\operatorname{Br}(T))<c^{+}$. The point of (iii) is that $\left\{\left(x_{\alpha}^{n}\right): n<\omega\right\}$ is neighborhood base for $b_{\alpha}$.

Let us determine the relevant cardinal functions for $B, L$, and $S$. The families $\left\{\left(x_{\alpha}^{n}\right): n<\omega\right\}$ demonstrate that $\chi(X)=\omega=\psi(X)$. A nonempty subset of $B$ contains some $(x)$, so by (i) $c(B)<c^{+}$. Because $B$ is a linearly ordered topological space, $s(B)=c(B)$. Because $B$ is dense in $L, c(L)=$ $c(B)$, so $c(L)<\omega_{2}$. Each $b_{\alpha}$ is a $G_{\delta}$ in $L$, so $c_{\omega}(L) \geqslant c^{+}$. Let $S=\omega \times L$. Every compact subset of $S$ is contained in some $m \times L, m<\omega$, so $K(S)=$ $\omega$. Next $c(S)=c(L) \cdot \omega<c^{+}$. We define open subsets $U_{\alpha}, \alpha<c^{+}$, of $S$ : $U_{\alpha}=\left\{\{n\} \times\left(x_{\alpha}^{n}\right): n<\omega\right\}$. Then $\left\{\left(\operatorname{int}_{\beta S} \mathrm{cl}_{\beta S} U_{\alpha}\right)-S: \alpha<\mathfrak{c}^{+}\right\}$is a disjoint family of nonempty open subsets of $\beta S-S$.

The existence of a space such as $A$ of $\mathrm{E}$ does not seem (at least to the author) to follow from the existence of a $c^{+}, \omega$ convenient tree. The results of $\$ 4$ also tend to indicate that $\mathrm{E}$ is fundamentally different from $\mathrm{A}-\mathrm{D}$. So we define $G_{\delta}$ tree families. For $\lambda, \mu$ regular, $\mu<\lambda$, a family $\mathcal{T}=\left\{T^{\alpha}: \alpha \leqslant \mu\right\}$ is a $\lambda, \mu G_{\delta}$ tree family iff

(i) $\forall \alpha \leqslant \mu T^{\alpha} \subset{ }^{\alpha} \lambda$;

(ii) $\forall \alpha<\mu T^{\alpha}$ is a $\lambda$ Souslin tree, $T^{\mu}$ is not;

(iii) $\forall \beta<\alpha<\mu \forall \sigma, \tau \in T^{\alpha} \sigma<_{T^{\alpha}} \tau$ implies $\sigma\left|\beta<_{T^{\beta}} \tau\right| \beta$; 
(iv) $x, y \in T^{\mu} X<T^{\mu} y$ iff $\forall \alpha<\mu x\left|\alpha<T^{\alpha} y\right| \alpha$.

We will construct the space $A$ from a c ${ }^{+}, \omega G_{\delta}$ tree family $\mathcal{T}$. Let $A$ be an antichain of $T^{\omega}$ of cardinality $\mathrm{c}^{+}$. The $n$th basic open neighborhood of $a$ is $B_{n}(a)=\left\{a^{\prime} \in A: a^{\prime}\left|n<_{n} a\right| n\right\}$. We note $e(A) \leqslant s(A) \leqslant c$, because $\mathrm{c}^{+}$is regular and each $T^{n}$ is a $c^{+}$Souslin tree. $A$ has a $G_{\delta}$ diagonal because for all $a \in A$

$$
\bigcap_{n<\omega}\left\{a^{\prime} \in A: a^{\prime}|n \leqslant a| n \text { or } a\left|n \leqslant a^{\prime}\right| n\right\}=\{a\} .
$$

3. In the previous section we constructed spaces from convenient trees and $G_{\delta}$ tree families. In this section we construct convenient trees and $G_{\delta}$ tree families.

Jensen $[\mathbf{J}]$ and Gregory $[\mathbf{G}]$ constructed $\omega_{2}$-Souslin trees from combinatorial principles. These trees are $\omega_{2}, \omega$ convenient trees as well. Not every $\omega_{2}$-Souslin tree is an $\omega_{2}, \omega$ convenient tree; using $\mathrm{CH}$ and $\diamond_{\omega_{1}}, \omega_{2}$ one can construct an $\omega_{2}$-Souslin tree "pruning" only at levels of cofinality $\omega_{1}$. However, we can construct a convenient tree from a Souslin tree.

THEOREM. If there is a $\lambda$-Souslin tree $S$, then there is a $\lambda, \omega$ convenient tree $T$.

Proof. As in the previous section, we may "squash" $S$ to an order complete line $L_{S}$. Because $L_{S}$ is order complete and dense, whenever $a<b$ there is $c, a<c \leqslant b$, such that $(a, c)$ has cofinality $\omega$.

Using this fact we will construct a new tree $T$ from $L_{S}$. Elements of $T$ will be intervals $(a, b)$; the order will be given by $(a, b)<\left(a^{\prime}, b^{\prime}\right)$ iff $(a, b) \supset$ $\left(a^{\prime}, b^{\prime}\right)$. By induction on $\alpha<\lambda$, we construct $T_{\alpha}$, the $\alpha$ th level of $T$ to satisfy the following conditions

0. $T_{0}=L_{S}$;

1. $\cup T_{\alpha}$ is dense in $L_{S}$;

2. every element of $T_{\alpha+1}$ is a proper subset of an element of $T_{\alpha}$ and has cofinality $\omega$;

3. every element of $T_{\alpha}$ contains infinitely many elements of $T_{\alpha+1}$;

4. if $(a, c) \in T_{\alpha}$ and has cofinality $\omega$, then for some $a^{\prime}\left(a^{\prime}, c\right) \in T_{\alpha+1}$;

5. for $\delta$ a limit ordinal $T_{\delta}$ is the set of intersections $\cap\left\{t_{\beta}: \beta<\delta\right\}$, where $t_{\beta} \in T_{\beta}$.

We verify that $T$ satisfies the three conditions to be a convenient tree. Because $S$ was a $\lambda$-Souslin tree, $T$ is, too, so $T$ satisfies (i). Condition 3 insures that $T$ satisfies (ii). By 4 and 5 , if $(a, c) \in T$ and has cofinality $\omega$, then the set of elements of $T$ with $c$ as endpoint determine a branch of cofinality $\omega$. By 2 , there are $\lambda$ such branches.

We now construct a $\lambda, \mu G_{\delta}$ tree family assuming $V=L$. There are two cases: $\lambda$ is strongly inaccessible and $\lambda=\kappa^{+}$. If $\lambda$ is strongly inaccessible, we use $E S \lambda$ : There are $E \subset \lambda, S=\left\{S_{\alpha}: \alpha \in E\right\}$ satisfying

(i) $E$ is stationary in $\lambda, \forall \alpha<\lambda$ there is $K_{\alpha}$ closed unbounded in $\alpha$, $K_{\alpha} \cap E=\varnothing$, and $\beta \in E$ implies $c f \beta=\mu$, 
(ii) $\forall X \subset \lambda,\left\{\alpha: S_{\alpha}=X \cap \alpha\right\}$ is stationary.

If $\lambda=\kappa^{+}$, we use $\square_{\kappa}^{*}$, which is $E S \kappa^{+}$plus the existence of $\left\{C_{\beta}: \beta<\lambda, \beta\right.$ a limit ordinal s satisfying

(iii) $C_{\beta}$ is closed and unbounded in $\beta$,

(iv) if $\gamma$ is a limit point of $C_{\beta}$ then $\gamma \notin E$ and $C_{\gamma}=C_{\beta} \cap \gamma$. Further, if $\mu>\omega$, we use $\kappa<\lambda, \nu<\mu$ implies $\lambda>\kappa^{\nu}$.

We will construct $T=T^{\mu}$ by constructing $T \mid \alpha$ and $A=\left\{a_{\beta}: \beta \in E\right\}$, the future large antichain, by induction on $\alpha<\lambda$. As we will use $S$ to fix mac's in each $T^{\nu}, \nu<\mu$, we assume, via a bijection between $\lambda$ and $U\left\{{ }^{\nu} \lambda: \nu<\mu\right\}$, that (ii) holds for $X \subset \cup\left\{{ }^{\nu} \lambda: \nu<\mu\right\}$. Our induction hypothesis on $\alpha$ will be

(1) $|T| \alpha \mid<\lambda, \forall \nu \leqslant \mu T^{\nu} \subset{ }^{\nu} \lambda$;

(2) $\forall \beta<\gamma<\alpha, \beta, \gamma \in E, o\left(a_{\beta}\right)=\beta, o\left(a_{\gamma}\right)=\gamma, a_{\beta} \nless a_{\gamma}$;

(3) $\forall \beta<\alpha \forall \nu<\mu S_{\beta}$ is a mac in $T^{\nu} \mid \beta$ implies $S_{\beta}$ is a mac in $T^{\nu} \mid \alpha$;

(4) $\forall \beta<\gamma<\alpha \forall x \in T_{\beta} \exists y \in T_{\gamma} x<y$;

(5) $\forall \beta<\gamma<\alpha \quad \forall \nu<\mu \quad \forall \sigma \in T_{\gamma} \quad \forall x \in T_{\beta}(x \mid \nu<\sigma$ and $\bar{x} \cap A=\varnothing)$ implies that (there are (at least) two distinct $y, z \in T_{\gamma} x<y, x<z,(\bar{y} \cup \bar{z})$ $\cap A=\varnothing, \sigma=y|\nu=z| v)$

(6) (i) (if $\lambda$ is inaccessible) $\forall \beta+1<\alpha, \beta \notin E$ every $\beta$-branch of $T \mid \beta$ continues;

(ii) (if $\left.\lambda=\kappa^{+}\right) \forall \beta+1<\alpha, \beta$ a limit ordinal $\beta \notin E$, if cf $\beta<\mu$ every $\beta$ branch of $T \mid \beta$ continues, if $\operatorname{cf} \beta \geqslant \mu$ the $\beta$ branch $b(x, \beta)$ continues, as defined below.

We define $b(x, \beta)$ by defining a set of $y$ 's cofinal in it. Let $<^{\prime}$ well order ${ }^{\mu} \lambda$. Let $\{\gamma(\delta): \delta<\eta\}$ be the monotone enumeration of $C_{\beta}$ (from $\left.\square_{\kappa}^{*}\right)$. Let $\bar{\delta}$ be least such that $x \in T_{\gamma(\bar{\delta})}$. Inductively define $y_{\delta}, \bar{\delta} \leqslant \delta<\eta$ :

$y_{\bar{\delta}}$ is the $<$ ' least $y \in T_{\gamma(\bar{\delta})}$ such that $x<y$ and $\bar{y} \cap A=\varnothing$ if $\bar{x} \cap A=\varnothing$. $y_{\delta+1}$ is the $<'$ least $y \in T_{\gamma(\gamma+1)}$ such that $x<y$ and $\bar{y} \cap A=\varnothing$ if $\bar{x} \cap A=\varnothing$.

$y_{\delta}$ for limit $\delta$ is the unique $y \in T_{\gamma(\delta)}$ such that $\bar{\delta} \leqslant \delta^{\prime}<\delta$ implies $y_{\delta}^{\prime}<y_{\delta}$. ( $y_{\delta}$ exists by (iv) and induction hypothesis.) This completes the definition of $b(x, \beta)$ and the statement of the induction hypothesis.

We construct $T \mid \alpha$ by induction on $\alpha$.

For $\alpha=1$ let $T_{1}$ be the constant function 0. For $\alpha$ a limit ordinal we must set $T \mid \alpha=\cup_{\beta<\alpha} T_{\beta}$. For $\alpha=\beta+1, \beta=\gamma+1$, give every element of $T_{\beta}$ two immediate successors and add more elements as required by (5).

If $\alpha=\beta+1, \beta$ a limit ordinal $\beta \notin E$, continue the branches as required by (6). If $\beta \in E$, continue only enough branches to satisfy (4) and (5). If $S_{\beta}$ is a mac in $T^{\nu} \mid \beta$, we require that these branches contain some $x, x \mid \nu \in S_{\beta}$. Then choose an element $a_{\beta} \in T_{\beta}$ to satisfy (2).

We verify that in this last case the construction can be done to satisfy the induction hypothesis, the other cases being simpler. By (i) let $\{\gamma(\delta): \delta<\eta\}$ be a monotone enumeration of a set $K_{\beta}$ closed, cofinal in $\beta, K_{\beta} \cap E=\varnothing$. Since $S_{\beta}$ is a mac in $T^{\nu} \mid \beta$ for every $x \in T \mid \beta$ there is $\sigma \in S_{\beta}, \sigma \leqslant x \mid \nu$ or $x \mid \nu<\sigma$. In the latter case by (5) there is $x^{\prime}, x<x^{\prime}, x^{\prime} \mid \nu=\sigma$ and $\bar{x}^{\prime} \cap A=\varnothing$ if $\bar{x} \cap A=\varnothing$. Call such an $x$ correct. For $x \subset T \mid \beta, x$ correct, let $\bar{\delta}$ be least 
such that $x \in T_{\gamma(\bar{\delta})}$. We may inductively choose $y_{\delta}, \bar{\delta} \leqslant \delta<\eta, y_{\delta} \in T_{\gamma(\delta)}$, $y_{\delta}>x$, using clause 4 of the induction hypothesis if $\delta$ is a successor, clause 6 if $\delta$ is a limit. Thus clause 4 of the induction hypothesis holds at $\alpha$. Clause 5 at $\alpha$ may be verified with the same technique. Having verified clause 5 at $\alpha$, we may use it to choose $a_{\beta}$ to satisfy clause 2 . Clause 5 continues to hold because we required two distinct elements for each $x, \sigma$.

By (2) $T$ has an antichain $A$ of cardinality $\lambda$. By (3) and the usual $\diamond$ argument, each $T^{\nu}, \nu<\mu$, is a $\lambda$ Souslin tree. This completes the construction of a $\lambda, \mu G_{\delta}$ tree family.

4. There are sup $=$ max-like questions associated with the inequalities $\mathrm{A}-\mathrm{E}$. For example, what is the class $C(\chi, c)$ of cardinal satisfying " $\chi(X)<\kappa$, $c_{a}(X)<\kappa$ implies $|X|<\kappa$ for Hausdorff $X$ ?"

(Associated with inequality $\mathrm{C}$ is the class $\mathrm{C}(\cdot, c)$ of cardinals $\kappa$ such that whenever $X$ is a compact $T_{2}$ space with $c_{a}(X)<\kappa$ then every disjoint family of intersections of $\nu<\kappa$ open subsets of $X$ has cardinality less than $\kappa$.)

THEOREM: (a) $\kappa$ weakly compact implies $\kappa \in \mathcal{C}(\chi, c) \cap C(d \psi, e)$;

(b) $\lambda<\kappa, 2^{\lambda} \geqslant \kappa$ implies $\kappa \notin \mathcal{C}(\chi, c) \cup \mathcal{C}(d \psi, e)$;

(c) $\kappa$ singular strong limit implies $\kappa \in C(\chi, c)-C(d \psi, e)$;

(d) $(V=L) \kappa$ inaccessible, not weakly compact implies $\kappa \notin \mathcal{C}(\chi, c) \cup$ $e(d \psi, e)$

(e) (a)-(d) hold with $C(\psi, s), \mathcal{C}(\cdot, c)$, or $\mathrm{C}(\kappa, c)$ in place of $\mathcal{C}(\chi, c)$.

Proof. (a) $\kappa \rightarrow(\kappa)_{\alpha}^{2}, \alpha<\kappa$.

(b) Consider $2^{\lambda}$ with the usual product topology.

(c) $\kappa \in \mathcal{C}(\chi, c)$ by [Ju, Chapter 3]. The space $\kappa+1$ where points $\alpha, \alpha<\kappa$ are isolated and the point $\kappa$ has the neighborhoods from the order topology shows $\kappa \notin \bigodot(d \psi, e)$.

(d) Consider convenient trees and $G_{\delta}$ tree families.

(e) As above. To show (b) for $\mathcal{C}(K, c)$, let $\lambda$ be least such that $2^{\lambda} \geqslant \kappa$.

Consider $\cup_{\alpha<\lambda}{ }^{\alpha} 2$ with the "tree" topology.

5. The question of whether the product of ccc spaces is ccc is an old question, but most of the results are recent. Kurepa $\left[\mathbf{K}_{\mathbf{1}}\right]$ showed that the product of two Souslin lines has cellularity $\omega_{1}$, and that the cellularity of a product of two (in fact, any family of) ccc spaces has cellularity at most $c$, $\left[\mathbf{K p}_{2}\right]$. This latter result can be proven by the Erdös-Rado theorem.

An interesting consequence of $\mathrm{MA}+\neg \mathrm{CH}$ is that the product of ccc spaces is ccc. Galvin [Ga] showed that $\mathrm{CH}$ allows the construction of two ccc spaces whose product is not ccc. Roitman [Ro] constructs a space similar to Galvin's in models obtained by adding a Cohen or random real. In this context Ketonen's question of whether $c(X)=\omega=c(Y)$ implies $c(X \times Y)$ $\leqslant \omega_{1}$ is natural.

In this section we show that adding $\kappa$ Cohen reals gives ccc spaces $X$ and $Y$ with $c(X \times Y) \geqslant \kappa$. We relabel the forcing conditions so that Galvin-type spaces are constructed directly from the generic filter. 
Let $P:[\kappa]^{2} \rightarrow\{0,1\}$. Let $X(Y)$ be the set of all $H \subset \kappa$ maximal with respect to $P^{\prime \prime}[H]^{2} \subset\{0\}\left(P^{\prime \prime}[H]^{2} \subset\{1\}\right)$. For $a \in[\kappa]^{<\omega}$, define $B_{X} a=\{H$ $\in X: a \subset H\}$ and $B_{Y} a=\{H \in Y: a \subset H\}$. Give $X(Y)$ the topology generated by $\left\{B_{X} a: a \in[\kappa]^{<\omega}\right\}\left(\left\{B_{Y} a: a \in[\kappa]^{<\omega}\right\}\right)$. It is easy to see that $c(X \times Y) \geqslant \kappa$, for $\mathcal{Q}=\left\{B_{X}\{\alpha\} \times B_{Y}\{\alpha\}: \alpha<\kappa\right\}$ is a family of disjoint nonempty open sets. $B_{X}\{\alpha\} \times B_{Y}\{\alpha\} \neq \varnothing$ because $[\{\alpha\}]^{2}=\varnothing$, hence $P^{\prime \prime}[\{\alpha\}]^{2}=\varnothing \subset\{0\} \cap\{1\}$. For $\alpha \neq \beta, P(\alpha, \beta)=0$, say, and then $B_{Y}\{\alpha\} \cap$ $B_{Y}\{\beta\}=\varnothing$, so $\mathcal{Q}$ is a disjoint family. The problem, thus, is to define $P$ so that $X$ and $Y$ are ccc. We accomplish this by defining $P$ generically.

Fix .k. Let $\mathscr{P}=\left\{p:|p|<\omega, p\right.$ is a funciton, dom $p \subset[\kappa]^{2}$, range $p \subset$ $\{0,1\}\}$. For $p \in \mathcal{P}$, define supp $p$ to be the smallest $a \subset \kappa$ such that dom $p \subset[a]^{2}$. Because later we will use fancier conditions we note that the proof below uses only that $\mathcal{P}$ is ccc and that $\operatorname{supp} p$ is finite.

Let $M$ be a countable transitive model of set theory, let $G$ be an $M$-generic ultrafilter on $\mathscr{P}$. Define $P:[\kappa]^{2} \rightarrow\{0,1\}$ by $P=\cup G$. Then if $X, Y$ are defined as above $c(X \times Y) \geqslant \kappa$. We show $X$ is ccc; of course, a parallel proof shows $Y$ is ccc.

If $X$ is not ccc then there is a family of $\omega_{1}$ disjoint nonempty open sets $B_{x} a$. We may apply the $\triangle$ system lemma to the $a$ 's and, because of the definition of $X$, throw away the common part. So assume that $p_{0} \in \mathcal{P}$, and

$$
\begin{gathered}
p_{0} \Vdash A=\left\{a_{\nu}: \nu<\omega_{1}\right\}, \quad \nu<\nu^{\prime}<\omega_{1} \rightarrow a_{\nu} \cap a_{\nu^{\prime}}=\varnothing, \\
B_{X} a_{\nu} \cap B_{X} a_{\nu^{\prime}}=\varnothing .
\end{gathered}
$$

For $n<\omega$, let $\mathscr{2}_{n} \subset \mathcal{P}$ be maximal with respect to

(a) $p \in \mathscr{Q}_{n} \rightarrow \exists e(p, n) \in M, p \vdash a_{n}=e(p, n)$,

(b) elements of $\mathcal{Q}_{n}$ are incompatible.

Let $F=\cup_{n<\omega, \mathscr{P} \in \mathcal{2}_{n}} e(p, n) \cup \operatorname{supp} p$. Because $\mathscr{P}$ is ccc, $\left|\mathscr{Q}_{n}\right|=\omega$, hence $|F|=\omega$. Note that $F \stackrel{M}{\in}$. By (*) there is $p_{1}<p_{0}, \nu<\omega_{1}, e_{1} \in M$

$$
p_{1} \Vdash a_{\nu}=e_{1}, \quad a_{\gamma} \cap F=\varnothing .
$$

Now because $\left|\operatorname{supp} p_{1}\right|<\omega$ we can extend $p_{1}$, perhaps $\left|\operatorname{supp} p_{1}\right|+1$ times, to get $p_{2}<p_{1}, n \in \omega, e_{2} \in M$, so that $\operatorname{dom} p \subset[F]^{2} \cup \operatorname{dom} p_{1}$ and

$$
p_{2} \Vdash a_{n}=e_{2}, \quad a_{n} \cap \operatorname{supp} p_{1}=\varnothing .
$$

Let dom $p_{3}=\left\{(\alpha, \beta): \alpha \in e_{1}, \beta \in e_{2}\right\}$, range $p_{3}=\{0\}$. Then

$$
p_{2} \cup p_{3} \Vdash B_{X}\left\{a_{v}\right\} \cap B_{X}\left\{a_{n}\right\} \neq \varnothing
$$

which contradicts (*), and establishes that $X$ is ccc.

This sort of generic construction is quite flexible. For example, we can change $\mathcal{P}$ so that the range of $\cup G$ is $\{m: m<n\}$. Set $X_{i}$ to be the set of $H \subset \kappa$ maximal with respect to $\cup G^{\prime \prime}[H]^{2} \subset\{m: m<n\}-\{i\}$. Then the product of any $n-1 X_{i}^{\prime}$ 's is ccc, proved as above, noting that the common value they all can use gives us room to work in. On the other hand, the open sets $\Pi_{i<n} B_{x_{i}}(\alpha), \alpha<\kappa$, show that $c\left(I_{i<n} X_{i}\right)=\kappa$. By similar fiddling around we can construct a space $W$ such that $c\left(W^{n}\right)=\omega_{n-1}$. 


\section{BIBLIOGRAPHY}

[De] K. Devlin, Aspects of constructibility, Lecture Notes in Math., vol. 354, Springer-Verlag, Berlin and New York, 1973.

[EH] P. Erdös and A. Hajnal, Unsolved problems in set theory, Proc. Sympos. Pure Math., vol. 13, Amer. Math. Soc., Providence, R.I., 1971, pp. 17-48.

[Ga] F. Galvin, Chain conditions and products, Fund. Math. (to appear).

$\left[\mathbf{G W}_{1}\right]$ J. Ginsburg and R. G. Woods, On the cellularity of $\beta X-X$, Proc. Amer. Math. Soc. 57 (1976), 151-154.

$\left[\mathbf{G W}_{2}\right]$

, A cardinal inequality for topological spaces involving closed discrete sets, Proc. Amer. Math. Soc. 64 (1977), 357-360.

[Gr] J. Gregory, Higher Souslin trees and the generalized continuum hypothesis, J. Symbolic Logic 41 (1976), 663-671.

[Je] R. Jensen, The fine structure of the constructible hierarchy, Ann. Math. Logic 4 (1972), 229-308.

[Ju] I. Juhász, Cardinal functions in topology, Math. Centrum, Amsterdam, 1971.

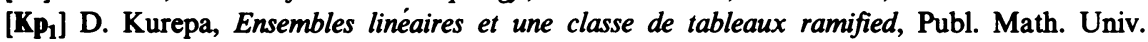
Belgrade 6 (1936), 129-160.

[K. $\left.\mathrm{P}_{2}\right]$, The Cartesian multiplication and cellularity numbers, Publ. Inst. Math. (Beograd) (NS) 2 (1962), 121-139.

[Ro] J. Roitman, Adding a Cohen or random real: Topological consequences and effect on Martin's axiom, Fund. Math. (to appear).

[Ru] M. E. Rudin, Souslin's conjecture, Amer. Math. Monthly 76 (1969), 1113-1119.

[Sh] B. Sapirovskii, Canonical sets and character. Density and weight in compact spaces, Soviet Math. Dokl. 15 (1974), 1282-1287.

Institute for Medicine aNd Mathematics, OHo University, ATHENS, OHIo 45701 